\title{
Cylinder Wipe Air-Drying Intaglio Ink Vehicles for U. S. Currency Inks
}

\section{Brian Dickens Barry J. Bauer William R. Blair}

\author{
U.S. DEPARTMENT OF COMMERCE \\ Natlonal Institute of Standards \\ and Technology \\ Materials Sclence and \\ EngIneering Laboratory \\ Polymers Division \\ Galthersburg, MD 20899

\section{Prepared for:} \\ The Office of Research and \\ Technlcal Services \\ Bureau of Engraving and Printing \\ Department of the Treasury \\ Washington, DC 20226
} NATONAL INSTIUTIE OF STANDARDS AND TECHNOLOGY John W. Lyons, Director 



\section{Cylinder Wipe Air-Drying Intaglio Ink Vehicles for U. S. Currency Inks}

\section{Brian Dickens Barry J. Bauer William R. Blair}

\author{
U.S. DEPARTMENT OF COMMERCE \\ Natlonal Institute of Standards \\ and Technology \\ Materlals Sclence and \\ EngIneerlng Laboratory \\ Polymers Divislon \\ Galthersburg, MD 20899
}

\section{Prepared for:}

The Offlce of Research and Technlcal Services

Bureau of Engraving and PrInting Department of the Treasury Washington, DC 20226

Annual Report of September 28, 1990 Issued January 1991

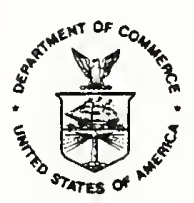

U.S. DEPARTMENT OF COMMERCE Robert A. Mosbacher, Secretary NATONAL INSTIUUTE OF STANDARDS AND TECHNOLOGY John W. Lyons, Director 



\section{Executive summary}

A family of air-dry intaglio resin alkyds has been developed. The alkyds are based on pentaerythritol, sebacic acid, linseed oil fatty acids and succinic anhydride. In laboratory tests, intaglio inks made from these resins washed well and cured to satisfactorily resistant films. Industrial quantities for press trials have been synthesized by a third party.

A second series of resins has also been developed. These resins are based on tripentaerythritol, linseed oil fatty acids and succinic anhydride. Satisfactory washing and solvent resistance are obtained in laboratory tests. Bulk quantities of this series are not available for press trials.

A third series of resins based on poly(vinyl alcohol) was synthesized but did not appear to be satisfactory for industrial development.

The temperature program is important in the synthesis of alkyds. The influence of temperature has been studied so that satisfactory resins can be made industrially in minimum time. 
Table of contents

1 Introduction

2 Alkyds based on pentaerythritol

2.1 Introduction

2.2 Alkyd 61

2.3 other pentaerythritol-based alkyds, including WB27

2.4 Varying acid number in second step of alkyd synthesis

2.5 Time/temperature in second step of alkyd synthesis

2.6 White solid in synthesized pentaerythritol alkyd.

2.7 Replacing sebacic acid with succinic anhydride . . . . . 13

3 Solvents in inks . • • . . • • • • • • • • • . . . . . 17

4 Super oil molecules based on tripentaerythritol . . . . . . 17

5 Drying molecules based on poly (vinyl alcohol) . . . . . . 20

6 Dielectric spectroscopy . . . . . . . . . . . . . . . 22

7 Microgels

8 References

Appendix: Synthesis of pentaerythritol alkyd 61

Table 1: Batches 1 to 4 of alkyd 61

Table 2: Batches 5 to 10 of alkyd 61

Table 3: Alkyds WB26 to WB31

Table 4: WB40 Alkyd synthesis and Characterization

Table 5: Alkyd Resin 27 Compositions . . . . . . . . . . . . . 12

Table 6: Tripentaerythritol-based Alkyds . . . . . . . . . . . 18

Figure 1: Alkyd viscosity versus solvent content . . . . . . . 8 Figure 2: SEC chromatograms of first step in synthesis . . . . 15 Figure 3: SEC chromatograms of second step in synthesis . . . . 16 
In the work covered by this Report, three types of air-dry resins ${ }^{1}$ were explored:

Type 1:

Type 1 is a high molecular weight, low-viscosity alkyd based on a polyester backbone of pentaerythritol and sebacic acid. This backbone is esterified with linseed oil fatty acids to confer the air-dry characteristics and with an acid anhydride to attach acid groups for dispersibility in Dalmar solution (aqueous $1 \%$ sodium hydroxide, 1\% surfactant solution).

Type 1 resins disperse easily in Dalmar solution. They air-dry well to give a reasonable degree of resistance to Dalmar solution, even though sensitivity to alkali is inherent in the structure of the resin.

Type 2:

Type 2 is a "super oil" molecule based on tripentaerythritol partially esterified with linseed oil fatty acids and acidified by reacting the remaining hydroxyl groups on the tripentaerythritol with an acid anhydride.

Type 2 resins were developed to provide liquid materials of low viscosity to which a minimum of solvent need be added. They disperse in Dalmar solution and give satisfactorily resistant films after drying.

Type 3:

Type 3 is a comb-type resin based on poly (vinyl alcohol) esterified with linseed oil fatty acids.

Type 3 resins are of low viscosity and air-dry rapidly, but are difficult to synthesize. Their dispersibility in Dalmar solution is not well characterized but in principle should not be problematic.

2 Alkyds based on pentaerythritol

\subsection{Introduction}

The philosophy in designing the family of alkyds based on pentaerythritol was that the backbone should be as flexible as feasible and there should be many fatty acid side chains to confer reasonable resistance to solvents after air-drying. The flexible diacid sebacic acid was used in the backbone.

Pentaerythritol was chosen as the polyol because it allows two 
side chains per polyol molecule, compared with only one side chain per molecule for the more traditional trimethylol propane. Air-dry capability was conferred by esterifying with linseed oil fatty acids. Dispersibility in Dalmar solution was conferred by using succinic anhydride to add carboxylic acid groups. Succinic anhydride leads to a much less viscous resin than do the more traditional phthalic and trimellitic anhydrides.

The synthesis of these alkyd resins consists of two steps:

Step One:

Linseed oil fatty acids, a diacid and a polyol are combined with xylene or decane solvent and refluxed until an acid number of approximately $10 \mathrm{mg} \mathrm{KOH} / \mathrm{g}$ resin is reached. The water from the esterification reaction is azeotroped off by solvent reflux at 170 to $210^{\circ} \mathrm{C}$, depending on the degree of reaction. At the end of the first step, the solvent is removed by distillation and the resin is cooled.

Step two:

Succinic anhydride is added at $120-125^{\circ} \mathrm{C}$. The resin is reacted with the anhydride at temperatures between $125^{\circ} \mathrm{C}$ and $180^{\circ} \mathrm{C}$ for half an hour.

The molecular weight of the resin increases as the esterification reaction proceeds. Such increase in molecular weight is known as chain extension. Some of the chain extension occurs in the first step of the reaction. The purpose of the second step of the reaction is to add acid groups to the resin, but chain extension continues in the second step to form yet higher molecular weight components in the resin using the free acid groups introduced in the second step of the alkyd synthesis.

The resin syntheses are carried out in the presence of solvent such as xylene to aid in removal of the water formed in the esterification step. Where a higher reaction temperature is needed, decane is used. The refluxing action of the solvent also serves to recover material which has splashed into the upper part of the reaction vessel. The reaction goes much more smoothly than in the absence of solvent and the amount of water caught in the Dean-stark side arm trap is more quantitative, which allows better tracking of the course of the esterification reaction. The solvent is removed by distillation at atmospheric pressure at the end of the first step, before the acid anhydride is added.

The progress in the esterification reaction has been followed using size exclusion chromatography. Size exclusion chromatography separates molecules based on their effective sizes. The effective size of a molecule depends on the solvent in which it is dissolved. The solvent most often used in the 
chromatograph is tetrahydrofuran, which is an excellent solvent for many organic materials. Both tetrahydrofuran and dichloroethane were used as chromatographic solvents.

Resins made from multifunctional reactants (e.g., diacids and trialcohols) contain distributions of molecular sizes which depend on the number of reactive groups per molecule, the amount of each ingredient used, and the degree of reaction attained. Size exclusion measurements show that the resins based on pentaerythritol typically contain high molecular weight material. The presence of this high molecular weight material may be one of the reasons that this type of resin is one of the best candidates for air-dry intaglio ink resins. Another reason is the higher degree of crosslinking possible in the cured film when a higher functional resin, i.e., one with more curable groups, is used. This is the reason given above for the choice of pentaerythritol as polyol.

\section{2 Alkyd 61}

In our earlier work ${ }^{2}$, an alkyd referred to as alkyd 61 was made using pentaerythritol, linseed oil fatty acids, sebacic acid and succinic anhydride. Inks formulated from alkyd 61 passed bench scale laboratory tests at BEP - an ink made from alkyd 61 washed with 8 to 10 strokes and cured to a solvent resistance of 3.5. (The standard ink washes with 8 to 10 strokes and has a resistance of 5 , where lower values indicate more resistance.) It therefore became desirable to scale up the synthesis to provide quantities which could be used on a small-scale research ink tester at BEP. Several syntheses were made at the 100 to 150 gram level. Reproducibility of the viscosity and acid number was good. A new reactor was then set up to make approximately $350 \mathrm{~g}$ of resin.

One kilogram of alkyd number 61 was synthesized in four batches (Table 1 ). The viscosities reported in Table 1 were determined with $a$ bob and cup viscometer. Residual solvent was estimated by heating a weighed sample $(0.3$ to $0.5 \mathrm{~g})$ of resin on a glass slide to $80^{\circ} \mathrm{C}$ for 2 to 3 hours.

Table 1 Batches 1 to 4 of alkyd 61

$\begin{array}{ccccc}\text { Prep \# } & \begin{array}{l}\text { 1st stage } \\ \text { acid number } \\ \text { mg KOH/g resin }\end{array} & \begin{array}{c}\text { final stage } \\ \text { mg KOH/g resin }\end{array} & \begin{array}{c}\text { viscosity } \\ \text { at } 30^{\circ} \mathrm{C} \\ \text { poises }\end{array} & \begin{array}{c}\text { residual } \\ \text { solvent }\end{array} \\ 1 & 8 & 77 & 120 & 0.03 \\ 2 & 13 & 80 & 70 & 0.18 \\ 3 & 8 & 86 & 75 & 0.06 \\ 4 & 12 & 75 & 50 & 0.08\end{array}$


In the first preparation, several anomalies were encountered. A stopper came loose during the first stage and a small but unknown amount of reactants was lost. The second stage reaction of the acid anhydride with remaining hydroxyl groups was unexpectedly exothermic and the temperature went too high.

Size exclusion measurements showed that the higher viscosity resins included some high molecular weight alkyd, as expected. The viscosity of a resin such as that obtained in preparation 1 can be adjusted by adding more solvent if the resin is too viscous or by adding higher molecular weight material if the resin is not viscous enough. We currently do not know the range over which such adjustments still provide acceptable ink resins.

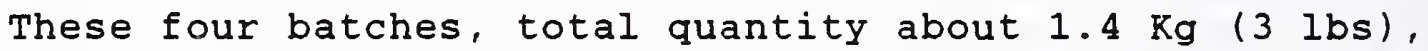
were taken to BEP and inks were formulated at BEP by Messrs. O'Brien and Weiss. Following a telephone report from Mr. O'Brien of BEP that these bench tests of alkyd 61 were promising, alkyd 61 was scaled up to provide about a gallon quantity (i.e., about $4 \mathrm{~kg}$ ) in batches of $700 \mathrm{~g}$. This work produced 6 batches, numbered 5 to 10 in Table 2, total quantity about $4 \mathrm{~kg}$ ( 1 gallon), with the properties summarized in Table 2 .

Table 2 Batches 5 to 10 of alkyd 61

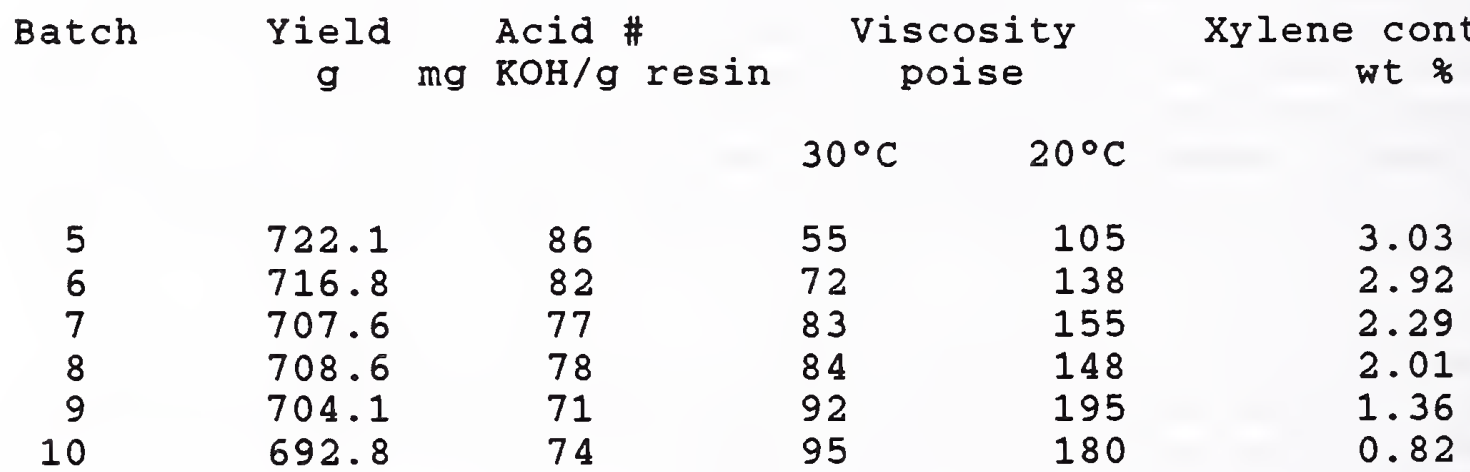

In addition to molecular weight, which should be about the same for all batches, the viscosity depends inversely on the solvent content, as shown in Figure 1 . 
Batches 1 to 10

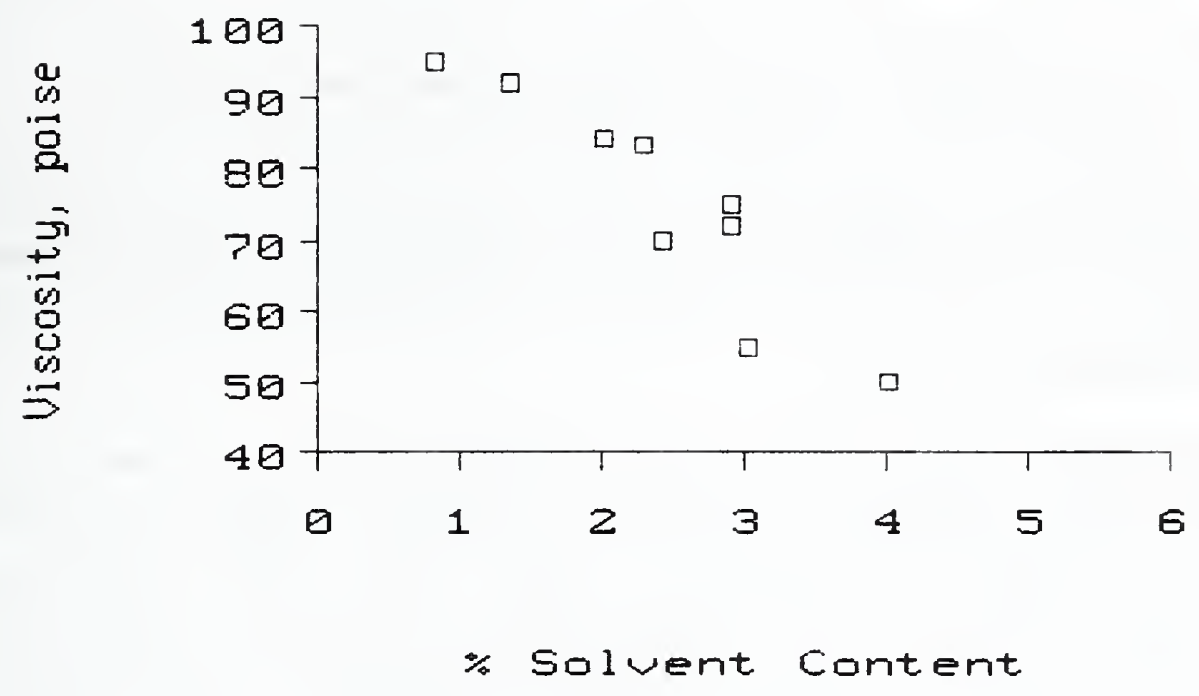

Figure 1. Viscosity at $30^{\circ} \mathrm{C}$ versus solvent content for alkyd preparations referred to in Tables 1 and 2 .

An ink was formulated at BEP from the alkyd 61 resins and tried on the research ink tester. The results of that

preliminary trial were promising. To test an intaglio resin on an operating press requires considerably more resin than we can produce. Therefore, at the request of Mr. O'Brien, we provided documentation (given in Appendix I) of the synthesis of the alkyd so that BEP could find a resin manufacturer who could produce for them the quantities they needed.

2.3 Other pentaerythritol-based alkyds, including WB27

At this point, the merit of alkyd 61 was well established in laboratory and small-scale tests. We investigated the possibility of fine-tuning the recipe to improve the washing performance in Dalmar solution. Six more alkyds (Table 3) based on the alkyd 61 recipe were synthesized. These alkyds had higher acid numbers. The acid number was increased in the following ways (the codes WB26, etc., refer to Table 3 ):

WB26: trimellitic anhydride was used instead of succinic anhydride to give two acid groups per anhydride molecule instead of the one acid group for every succinic anhydride molecule reacted. It was then possible to use more linseed oil fatty acids (LOFA). This resin was very viscous, as expected when an aromatic group is introduced into the alkyd molecule. 
WB27: sebacic acid and LOFA were partially replaced with succinic anhydride, which gave a resin of higher acid number and somewhat higher viscosity (more hydrogen bonds, less LOFA) than alkyd 61 .

WB28: some of the LOFA in alkyd 61 was replaced with succinic anhydride, which increases the viscosity of the resultant resin more than for WB27 and alkyd 61.

WB29: a mixture of equal amounts of pentaerythritol (PE) and dipentaerythritol (DiPE) was used. Less sebacic acid than for alkyd 61 was required in this case because some of the polyol linking done by the sebacic acid was already present in the dipentaerythritol.

WB 30 and WB31 contain less sebacic acid than the other members of the series, which gives smaller alkyd molecules and hence less viscous resins.

Table 3 is a comparison of the six alkyds.

$$
\text { Table } 3 \text { Alkyds WB26 to WB31 }
$$

Alkyd ID:

Residual solvent, $\%$

Acid Number, mg ROH/gram

Viscosity at $20 \mathrm{C}$, poises

Viscosity at $30 \mathrm{C}$, poises

Strokes to wash

Dalmar resistance

(from strokes)
WB26 WB27 พB28 WB29 พB30 พB31

$\begin{array}{llllll}3.2 & 0.8 & 0.6 & 0.1 & 0.1 & 0.2\end{array}$

$\begin{array}{llllll}97 & 99 & 103 & 98 & 90 & 93\end{array}$

$\begin{array}{llllll}-- & 300 & 500 & 680 & 105 & 65\end{array}$

$\begin{array}{lllll}-- & 148 & 310 & 400 & 52\end{array}$

all in the range of 4 to 7 strokes

$\begin{array}{lllll}4 & 3 & 3.5 & 5 & 4.5\end{array}$

(Dalmar resistance of alkyd 61: 3.5 )

The molar content of each recipe was:

$\begin{array}{lccccccc}\text { Alkyd ID: } & \text { WB26 } & \text { WB27 } & \text { WB28 } & \text { WB29 } & \text { WB30 } & \text { WB31 } & 61 \\ \text { LOFA } & 1.7 & 1.4 & 1.25 & 1.8 & 1.6 & 1.7 & 1.5 \\ \text { PE } & 1.0 & 1.0 & 1.0 & .50 & 1.0 & 1.0 & 1.0 \\ \text { DiPE } & - & - & - & .50 & - & - & - \\ \text { Sebacic acid } & .55 & .40 & 0.55 & .30 & .30 & 0.25 & .55 \\ \text { Succinic anhydride } & - & 1.35 & 1.25 & 1.80 & 1.35 & 1.35 & 1.0 \\ \text { Trimellitic anhydride } & .80 & - & - & - & - & - & -\end{array}$


The main feature of all these new variations on the theme of alkyd 61 is that the acid number was increased to 100 in various. ways. Laboratory tests at BEP on inks formulated from these alkyds showed that all washed well in Dalmar solution (4 to 7 strokes) and had adequate cure resistance ( 3.5 to 5) to Dalmar solution after one week of air-drying.

WB27 was scaled up to 55 gallons by a third party. Press trials have not yet been carried out. WB27 is of intermediate viscosity compared with the other alkyds in Table 3. The same viscosity but different cure properties would be obtained by mixing (WB28 and/or WB29) with (WB30 and/or WB31).

2.4 Varying acid number in second step of alkyd synthesis

Six alkyds were made, designated the WB40 series, all having the same first step, but with different amounts of succinic anhydride. The syntheses used the same first step as in alkyd WB27 with the reactants scaled up to $500 \mathrm{~g}$ total weight. The second step was carried out in stages at about $125^{\circ} \mathrm{C}$.

Approximately $100 \mathrm{mls}$ were removed at each stage and more anhydride was added. In this way we produced a series of alkyds that vary only in acid number (Table 4). The two highest amounts of succinic anhydride produced samples with a second insoluble phase, probably due to unreacted anhydride. All these samples were taken to BEP for ink formulation and testing.

Table 4 WB40 Alkyd Synthesis and Characterization

Step 1

Linseed oil fatty acids

Sebacic acid

Pentaerythritol

XYlene

$$
\begin{aligned}
& 321.31 \mathrm{~g} \\
& 66.73 \mathrm{~g} \\
& 111.96 \mathrm{~g} \\
& 50 \mathrm{~g}
\end{aligned}
$$

Step 2

Sample Succinic Removed Acid Number Viscosity Phases

$\begin{array}{lccrrrr}\text { I } & - & 90.2 \mathrm{~g} & 9 \mathrm{mgKOH} / \mathrm{g} & 7 & \text { poise } & 1 \\ \text { II } & 29.53 & \mathrm{~g} & 91.7 & 43 & 10 & 1 \\ \text { III } & 22.65 & 94.4 & 84 & 20 & 1 \\ \text { IV } & 16.22 & 96.5 & 94 & 34 & 1 \\ \text { V } & 10.18 & 96.0 & 113 & 47 & 2 \\ \text { VI } & 4.48 & - & 141 & 80 & 2\end{array}$


The column headed "Succinic" indicates the amount of additional succinic anhydride added at this stage. "Removed" is the amount of sample removed at each stage. "Phases" gives the number of components in the final alkyd, 2 indicating that a solid white phase was present in addition to the liquid resin.

Size exclusion chromatography showed that these alkyds were much less extended to high molecular weight material than alkyds 61 and WB27, as indicated by their low viscosities (Table 4). The main difference from earlier syntheses was that the second step had been carried out at $125^{\circ} \mathrm{C}$. Thus, it appeared that appreciable chain extension occurred when the second step was carried out at $180^{\circ} \mathrm{C}$ but not when it was carried out at $125^{\circ} \mathrm{C}$. This finding prompted an examination of the time/temperature dependence of the second step of the synthesis.

\subsection{Time/temperature in second step of synthesis}

Using the formula for alkyd WB27 as a starting point, several two-step synthesis reactions incorporating modified quantities of reactants or changes in procedure were performed. The goal was to examine the influence of reactant ratios and reaction temperatures on the resin product. This provides further understanding and control of the reaction to aid in the design of a resin with the properties needed to produce a good air-drying ink resin.

In a second experiment (alkyd 42), step one of the synthesis for WB27 was carried out until an acid number of $<10 \mathrm{mg} \mathrm{KOH} / \mathrm{g}$ resin was reached. Additional aliquots of sebacic acid were added as the reaction was continued. A total of five sebacic acid additions were made, each contributing an additional 5\% more sebacic acid to the resin. Samples for SEC analysis were withdrawn every thirty minutes. The SEC results (Figure 2) show a steady increase in high molecular weight components of the resin as each additional amount of sebacic acid was added to the resin.

In a re-examination of step two of the alkyd synthesis, step one was scaled up to yield approximately $270 \mathrm{~g}$ of product. Upon the completion of step one, solvent was removed by distillation and the resin cooled and divided into two equal portions. Each portion of the resin was finished by a different step two procedure. In the first case, succinic anhydride was added and reacted at $180^{\circ} \mathrm{C}$, with samples being withdrawn every ten minutes, until the resin gelled. In the second case, succinic anhydride was added and reacted at $125^{\circ} \mathrm{C}$, with samples being withdrawn initially at ten, then at twenty and finally thirty minute intervals for a period of three hours. This synthesis was stopped before any gelling of the resin was observed (Figure 3 ). 
WB-44, Varied Sebacic Acid Content

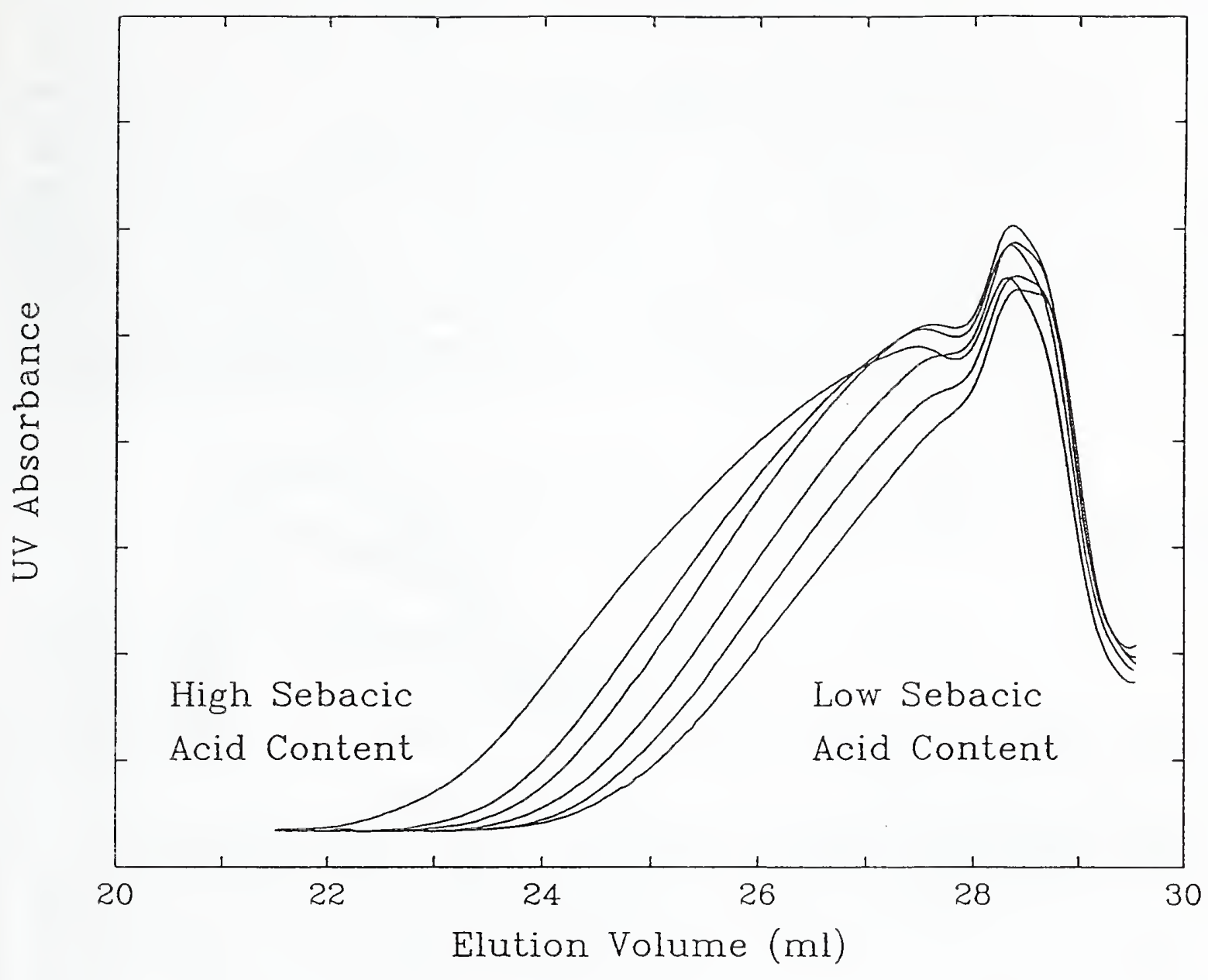

Figure 2: SEC chromatograms, with UV detector response set at a wavelength of $254 \mathrm{~nm}$, for alkyd resin samples with increasing amounts of sebacic acid in the first step of the synthesis. The elution volume abscissa corresponds to a molecular weight gradation with high molecular weight on the left and low molecular weight on the right. The curves from right to left correspond to increasing sebacic acid content. 


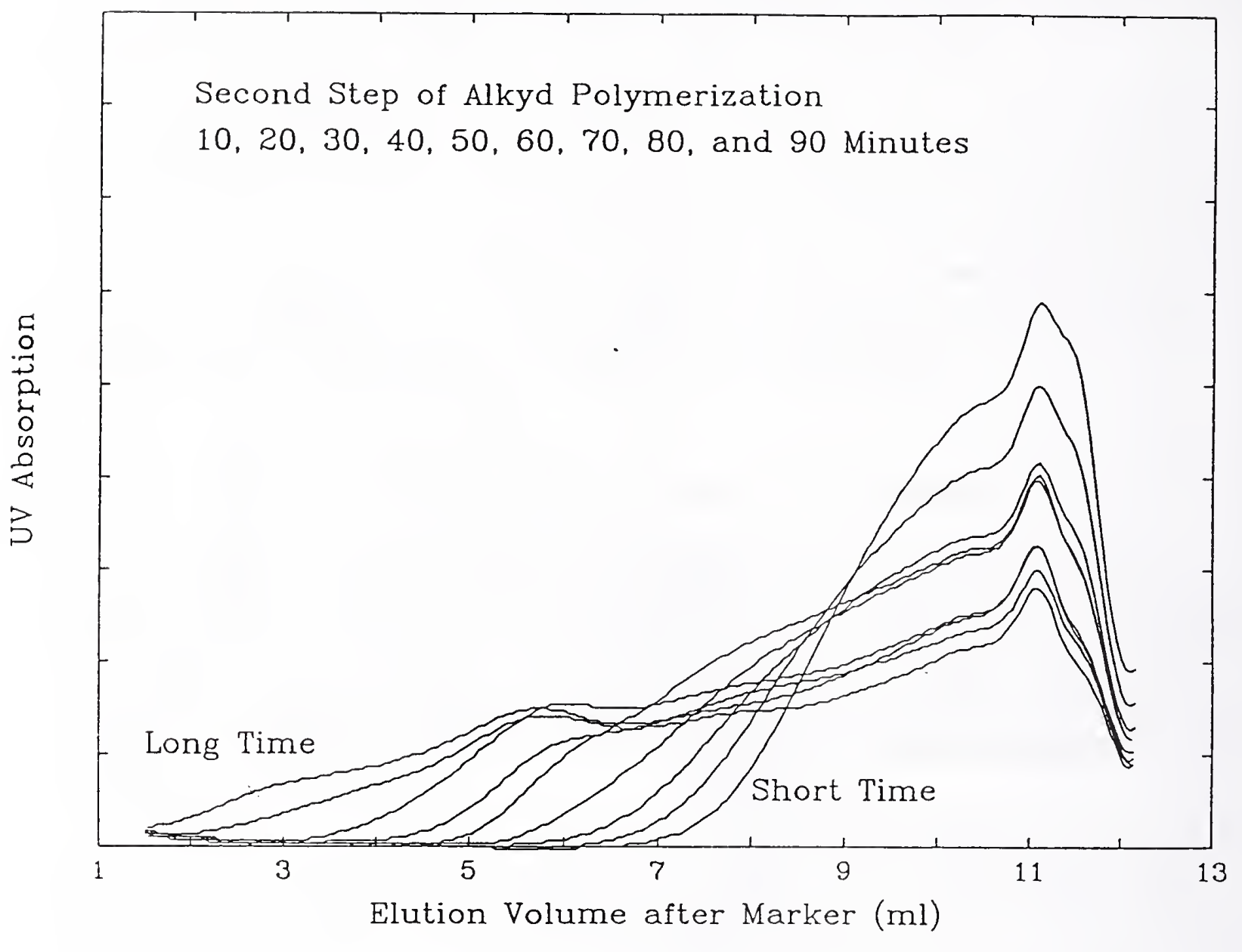

Figure 3: SEC chromatograms, with UV detector response set at a wavelength of $254 \mathrm{~nm}$, for samples removed from step two of synthesis of alkyd WB42. The elution volume abscissa corresponds to a molecular weight gradation with high molecular weight on the left and low molecular weight on the right. Samples were removed at $10,30,50,70$ and 90 minute intervals, with zero time being the time at which the reaction mixture reached $180^{\circ} \mathrm{C}$ following the addition of succinic anhydride. The 10 minute sample is the right-most curve, rising from baseline at an elution volume of approximately $7 \mathrm{~mL}$. The curve second from right, is the 30 minute sample, rising from baseline just past the $6 \mathrm{~mL}$ elution volume point. The 30 through 90 minute sample curves are shown sequentially from right to left. The resin became too thick to sample by pipette after 100 minutes. 
Based on the above results, the formula for alkyd WB 27 was modified (Table 5) to include $25 \%$ more chain extending diacid (sebacic acid) to promote additional chain extension in step one of the synthesis. Step one components were reacted to an acid number of $<10 \mathrm{mg} \mathrm{KOH} / \mathrm{g}$ resin, solvent was removed and succinic anhydride added at $125^{\circ} \mathrm{C}$. The second step of the reaction was continued for one hour at about $125^{\circ} \mathrm{C}$, at which point the resin was cooled and poured into a jar for storage. Some gelled material was noted in the resin as it was poured into the storage jar, indicating that step two may have carried out for too long a time, even though the temperature was only $125^{\circ} \mathrm{C}$. Analysis of this resin by SEC at the end of step one and at the end of step two showed that the molecular weight of the soluble part of the resin decreased between step one and two, due to the removal of high molecular weight components by gellation.

\section{Table 5 Alkyd Resin 27 Compositions, g}

Linseed Oil Sebacic Pentaerythritol Fatty Acids Acid

Alkyd WB 27

86.67

86.67
18.0

30.28

22.5

30.28
Modified

Alkyd WB 27
Succinic Anhydride

30.1

The above work demonstrates a relationship between the time to gel and the reaction temperature in the second step. For minimum time, the reaction should be carried out at the highest temperature at which it can be controlled. Alkyd 27 was successfully made with a temperature of $180^{\circ} \mathrm{C}$ in the second step. This is probably as high a temperature as is as feasible for meaningful control of the extent of reaction because free acid groups esterify at 170 to $210^{\circ} \mathrm{C}$, as in the first step of the synthesis. The SEC analyses in Figure 3 show that the molecular weight distribution of the resultant alkyd depends on the temperature program used in the resin synthesis. Thus, optimizing the alkyd resin includes optimizing the combination of ingredients and also optimizing the reaction temperature program.

2.6 White solid in pentaerythritol alkyd

A white solid was noticed in WB27 and in the industrially made equivalent, SVD 1781. This solid was tentatively identified as succinic acid as follows:

From the reactants used, the solid could reasonably be expected to be pentaerythritol, sebacic acid, succinic anhydride, or succinic acid. Pentaerythritol does not dissolve in acetone, the others do. The white solid was shown to be soluble in acetone. 
A sample of resin containing the white solid was heated between microscope cover glasses on an electrically controlled melting point apparatus. The solid disappeared at $180-190^{\circ} \mathrm{C}$. sebacic acid melts at $138^{\circ} \mathrm{C}$. Succinic acid melts at $188^{\circ} \mathrm{C}$. Succinic anhydride melts at $120^{\circ} \mathrm{C}$. Pentaerythritol melts at $257^{\circ} \mathrm{C}$. A specimen of sebacic acid placed on the cover glass melted as expected and showed that the temperature was being measured correctly. A specimen of succinic acid placed on the cover glass formed long needles. As the resin cooled, the solid in the resin reappeared as long needles, the crystalline form expected for succinic acid. A little succinic acid was crushed into a new specimen of resin containing the white powder. Both the succinic acid and the white powder disappeared together on heating, some small allowance being made for the larger granular size of the crushed material. The succinic acid presumably arises when the succinic anhydride used in the final step reacts with water remaining from the first step of the synthesis. Succinic acid will not react further at the temperature used. Therefore any succinic acid remains unreacted.

\subsection{Replacing sebacic acid with succinic anhydride}

Investigations were carried out to attempt to reduce the two step alkyd synthesis to a one step procedure with succinic anhydride being used in both steps one and two. Alkyd syntheses were carried out using succinic acid or anhydride with linseed oil fatty acids and pentaerythritol in the first step. These components were similar to those used for previous alkyds except that the diacid chain extender was succinic acid rather than sebacic acid. The formula for alkyd WB27 was modified by substituting $11.0 \mathrm{~g}$ of succinic acid for $18.0 \mathrm{~g}$ of sebacic acid. In the first stage of the synthesis, the ingredients were heated with xylene at temperatures up to $210^{\circ} \mathrm{C}$, driving off xylene and water until the acid number reached $10 \mathrm{mg} \mathrm{KOH} / \mathrm{g}$ resin. Succinic anhydride was then added in the second stage to increase the acid number.

When succinic acid was used in the first step, a large amount of undissolved white material remained. It appeared to be unreacted succinic acid, appearing as the same small spheres that were originally added. The high melting point and low solubility of the diacid in the other ingredients prevent it from participating in the reaction.

The experiment was repeated with succinic anhydride replacing the acid in equal molar amount. Having a lower melting point and being more reactive, the anhydride should react more than the acid under these conditions. After the first stage, however, a sizable amount of white insoluble powder remained. 
the starting ingredients contain water, the first reaction will be the formation of the acid from the anhydride which would cause the same problems as before.

A three-step synthesis procedure using the modified formula for alkyd WB27 was designed to attempt to react all the succinic acid. The three-step synthesis was as follows:

Step one: Combine the linseed oil fatty acids and the polyol (pentaerythritol) with xylene and react at 205 to $230^{\circ} \mathrm{C}$ until an acid number less than $10 \mathrm{mg} \mathrm{KOH} / \mathrm{g}$ resin is obtained.

Step two: Cool the reactants to $160^{\circ} \mathrm{C}$ and add succinic acid. Increase the temperature until the xylene solvent refluxes at $195^{\circ} \mathrm{C}$. Continue heating until the acid number again drops to below 10 .

Step three: Cool the resin and add succinic anhydride. React for an additional half hour at $125^{\circ} \mathrm{C}$.

When the three-step synthesis was performed, undissolved succinic acid was noticed in the reaction flask during step two when samples were removed for acid number determinations. In step two, the refluxing of xylene limited the reaction temperature to a maximum of $195^{\circ} \mathrm{C}$. It was felt that this temperature was close enough to the $187-189^{\circ} \mathrm{C}$ melting point temperature of succinic acid that it may have been limiting the degree of succinic acid reaction. An additional modification of the synthesis procedure was tried at this point in an attempt to dissolve and react all the succinic acid. The xylene was removed from the reaction flask and the reaction temperature increased to $240^{\circ} \mathrm{C}$ with the expectation that all the succinic acid would react. After two hours and forty minutes at $240^{\circ} \mathrm{C}$, unreacted succinic acid continued to remain in the reaction flask. At this point the reaction was abandoned and the third step was not carried out.

One additional synthesis scheme was designed in an attempt to make an alkyd resin with succinic acid as diacid. A solventless procedure was used, the reaction being carried out at 210 to $240^{\circ} \mathrm{C}$ with a nitrogen gas purge to remove water formed in the first step esterification reaction of linseed oil fatty acids and polyol. This scheme proved impractical as the nitrogen gas purge, though successful in removing water formed by the reaction, also removed reactants from the flask and deposited them throughout the water trap and condenser assembly.

Succinic acid was used in these syntheses to allow us to adapt the typical two-step procedure we have used with the sebacic acid-based alkyds to the use of succinic anhydride in both the first and second steps, thus reducing the synthesis to one step. The succinic anhydride was to replace the sebacic acid. Succinic acid was used instead of succinic anhydride as an 
intermediate case. The results with succinic acid suggest that its high melting point makes it too difficult to work with in these syntheses and this path was therefore abandoned.

A problem arose when it was found that inks based on several months old samples of $\mathrm{WB} 27$ and a duplicate resin from an industrial source did not disperse well in Dalmar solution. Also, an ink made several months earlier from WB27 no longer dispersed well. These inks took about 20 strokes to disperse. However, it was found that the washing could be greatly improved by adding mineral spirits solvent to the inks.

This suggests an important and probably already well known principle in the process of formulating an ink. The ink can be formulated conceptually without considering the amount of solvent to be added. That is, the quantity of resin is selected so that it covers the solid materials and enough drier is used to cure the resin. These liquids and the various solids are taken to represent $100 \%$.

Solvent can then be added to this mixture in any amount in principle. In practice, the amount is limited by air pollution regulations and the desired application viscosity of the ink. The solvent amount is to a first approximation irrelevant to the formulation of the ink because the solvent will evaporate as the ink cures.

For reasonably small amounts of solvent (up to $12 \%$ of all other ingredients combined is the maximum allowed by Air Quality Regulations ${ }^{3}$, any initial amount of solvent will lead to the same cured ink. The viscosity of the ink without solvent must therefore be such that the desired application viscosity can be attained using $12 \%$ or less of solvent, giving a maximum "content" of $112 \%$ in that case.

\section{Super oil molecules based on tripentaerythritol}

Alkyds 15 to 53, made in FY88, were based on tripentaerythritol. A new alkyd, WB32, based on tripentaerythritol was synthesized in the work described here. The acid number was designed to be 100. The acid number was found to be 68 , which, being lower than the expected 100 , showed that the carboxylic acid group formed by the anhydride addition underwent a considerable amount of further esterification to give unwanted crosslinking. The anhydride had been added at $155^{\circ} \mathrm{C}$ instead of $125^{\circ} \mathrm{C}$. An ink made from WB32 cured very rapidly, as expected from the apparently higher than expected degree of crosslinking in the parent resin, but required 11 to 14 strokes to disperse in Dalmar solution because of the low acid number. 
Alkyds WB34, WB35, WB35II, WB36 and WB37 were then synthesized to explore the feasibility of increasing the acid numbers of tripentaerythritol-based alkyds. During the preparation of alkyd WB35, the temperature controller malfunctioned and the temperature went too high. clumps of tripentaerythritol formed and the stoichiometry of the reaction became unknown. WB35II is a repeat of WB35. Details of the alkyds are given in Table 6:

Table 6: Tripentaerythritol-based Alkyds

alkyd

Molar composition:

Tripentaerythritol

Linseed oil fatty acids

succinic anhydride

Intended acid number

Solvent

( $x=x y$ lene, $d=$ decane)

Final acid number

Viscosity at $20 \mathrm{C}$, poises

Viscosity at $30 \mathrm{C}$

* Residual solvent

Appearance after stage 1:clear

Appearance after stage 2:clear clear

Strokes to wash

(minutes to clean slide)

(standard ink: 2:30-3:50)

Emulsion quality

Dalmar resistance

(standard ink: 5)
WB32 WB34 WB35 WB35II WB36 WB37

$\begin{array}{rrrrrr}1 & 1 & 1 & 1 & 1 & 1 \\ 3.9 & 3.9 & 3.8 & 3.8 & 3.75 & 3.75 \\ 3.2 & 3.2 & 3.5 & 3.5 & 3.75 & 3.75 \\ 100 & 100 & 110 & 110 & 117 & 117\end{array}$

$\begin{array}{llllll}x & d & d & d & d & d\end{array}$

$\begin{array}{llllll}68 & 76 & 113 & 98 & 144 & 121\end{array}$

$\begin{array}{rrrrrr}155 & 400 & 140 & 320 & 56 & 200 \\ 75 & 200 & 64 & 170 & 32 & 129\end{array}$

$\begin{array}{llllll}0.1 & 1.8 & 3.4 & 1.3 & 3.9 & 4.7\end{array}$

$\begin{array}{lrrr}* & 6-7 & 6-8 & 15-16 \\ * & 1: 58 & 1: 46\end{array}$ * $\quad$.

* fair good good * good

* $2.5 \quad 5.5 \quad 5.5 \quad 6$

$\star=$ not tested

Problems were encountered in both steps of the syntheses. Tripentaerythritol melts at a higher temperature than does pentaerythritol. This means that the esterification reaction between tripentaerythritol and linseed oil fatty acids must be carried out at higher temperature than the esterification reaction involving pentaerythritol. In the case of alkyds based on pentaerythritol, xylene is refluxed to azeotrope water off 
from the esterifying mixture. involving tripentaerythritol, agent because it has a higher xylene, decane is hydrophobic and can therefore be
azeotrope water off from esterification reactions.
In several of these preparations decane was used as the refluxing boiling point than xylene. Like and can therefore be used to

That part of the tripentaerythritol which reacts in the early stage of the reaction is expected to become fully esterified, because at that point there is a large excess of linseed oil fatty acid in the liquid phase. If only part of the tripentaerythritol dissolved in the first step of the synthesis, then most or all of the hydroxyl groups on the dissolved tripentaerythritol would have been consumed in the esterification step, leaving no hydroxyl groups for the anhydride addition step. To even out the degree of esterification of the tripentaerythritol by transesterification, WB37 was held at the esterification temperature for much longer than the other alkyds and the reaction was carried out in the presence of the esterification catalyst p-toluene sulfonic acid.

The acid anhydride reacted anomalously with the product from the esterification step. We were aiming at acid numbers of 100 to 120 , which require an unusually large amount of acid anhydride. If the temperature is allowed to go much above $130^{\circ} \mathrm{C}$ during the anhydride addition, the acid groups attached to the alkyd in the anhydride reaction react further with unreacted hydroxyl groups on other alkyd molecules and cause crosslinking to occur.

The indications of the course the reaction followed are:

1) If the reaction product is cloudy, not all the tripentaerythritol dissolved and what did dissolve was mostly consumed by the linseed oil fatty acids, leaving few hydroxyl groups to react with the acid anhydride. Thus, the viscosity of the resin will be low and the final acid number of the mixture will be higher than intended, as was observed in resins WB35 and WB36.

2) If the reaction product is clear and the acid number is too low, unwanted esterification of the acid groups derived from the anhydride addition occurred. The resin will be more viscous than intended and will probably cure well, but will not disperse well in Dalmar solution, as found for resins WB32 and WB34.

3) If the reaction product is clear and the acid number is too high, the acid anhydride did not react as much as intended, and the resin will not disperse in Dalmar solution as well as intended, as in the case of resin WB37.

Inks were made from these alkyds at BEP. Most of the inks washed well ( 8 strokes or less). The resistance of the inks made with resins with higher acids numbers was not better than that of 
the standard ink. The resistance of the ink based on WB34 was significantly better than that of the standard ink. WB34 should be considered for scaleup and press trials.

Drying molecules based on poly (vinyl alcohol)

The alkyd approach to air-dry inks provides resins and inks which dry fairly slowly and which need Dalmar solution to be dispersed from the wiping mechanism. We explored the feasibility of using derivatives of polyvinyl alcohol to provide other possibilities for air-dry resins. Polyvinyl alcohol (PVA) is prepared by hydrolyzing polyvinyl acetate. The hydrolysis is not carried out to completion because the product is then so hydrogen-bonded that it is insoluble.

2000 MW 75\% hydrolyzed poly vinyl acetate was used. This means that the average polyvinyl alcohol molecule has about $35 \mathrm{OH}$ groups, which in principle could be reacted with linseed oil fatty acids (LOFA) to give 35 fatty acid side chains. Thus, $2 \mathrm{~g}$ of polyvinyl alcohol require about $10 \mathrm{~g}$ of linseed oil fatty acid for complete reaction of all the hydroxyl groups. If all the $O H$ groups were reacted, there would be no solubilizing mechanism. If insufficient $O H$ groups are reacted, there is insufficient air-dry mechanism. If there are insufficient oH groups left to provide a solubilizing mechanism after enough air-dry capability has been built into the molecule, pendant acid groups could be introduced by reacting acid anhydrides with residual oH groups as is done in the case of alkyds.

A synthesis was carried out using polyvinyl alcohol and linseed oil fatty acids in the ratio $3: 2$ and xylene as a refluxing solvent, as is often done in the preparation of alkyds. The intent was to react about $15 \%$ of the oH groups. Roughly twice the expected amount of water was obtained, which suggested that either etherification between PVA molecules or double bond formation along the PVA backbone may have been occurring. Water absorbed in the PVA, which is hydrophilic, was also included. The reaction product was a solid partially dispersible and partially insoluble in water, which suggests that some crosslinking etherification reactions had occurred (this is a lower temperature reaction than elimination of water from secondary hydroxyl groups). In a second attempt, the reaction was carried out in DMF using a PVA:LOFA ratio of $2: 1$ (equivalent to esterifying about $10 \%$ of the OH groups). A pasty solid, completely dispersible in water, was obtained.

Several syntheses were then carried out. They fall into two categories. The first category is an alkyd-like esterification reaction. In this type of reaction, temperatures can easily reach over $200^{\circ} \mathrm{C}$. PVA is a somewhat unstable solid, melting at about $170^{\circ} \mathrm{C}$ and undergoing decomposition during prolonged heating. At these temperatures, the unwanted side reaction of 
water elimination by double bond formation along the PVA backbone can become significant. Two solvents were tried. The first was xylene, which is used in alkyd syntheses to carry off the water evolved during esterification. PVA is not soluble in xylene. Since PVA is soluble in dimethyl formamide (DMF), we also used DMF as solvent in several trial syntheses. However, problems arose in removing the water of esterification from the DMF and also in removing the DMF from the reaction products.

During these esterifications, the reaction mixture became much more viscous as liquid free fatty acid was used up. Many of the syntheses were intended to produce incompletely esterified PVA so that remaining alcohol groups could be reacted with acid anhydrides if necessary. These reaction products were pasty solids. To examine the final form of fully esterified PVA, we carried out the reaction with a large excess of linseed oil fatty acid. A one phase liquid, mostly unreacted fatty acid which was present in great excess, was produced. The reaction products were examined by size exclusion chromatography. It proved to be very difficult to find the reaction products in the resultant refractive index detector chromatogram, probably because the refractive index of partially esterified PVA is close to that of the solvent usually used in size exclusion chromatography. However, some indication of a completely esterified PVA was obtained. The chromatographic runs were repeated using an evaporative mass detector, which clearly showed that large molecules are present in the reaction mixture and allowed us to conclude that the reactions did indeed proceed to completion. This technique was then used routinely to monitor extents of reaction in our PVA/LOFA syntheses.

The second category of reaction was to esterify PVA using the acid chloride of linolenic acid to produce the intended end product conveniently so that its properties could be assessed. The hydrogen chloride liberated in the reaction was taken up with polyvinyl pyridine, which was then filtered off. Complete esterification of the PVA by the acid chloride requires weight ratios of 1:5.3 PVA:LC (Iinolenoyl chloride). We made two successful preparations using DMF as solvent:

weight ratio
1) 1 PVA
2) 1 PVA
$2.5 \mathrm{LC}$
1.5 LC
(linolenoyl chloride)

The reaction products are brown liquids with viscosities in the range of 100 poises (estimated, not measured) which skin over easily and thus air dry quickly. They may be emulsifiable in Dalmar solution in their current forms. If not, some of the remaining hydroxyl groups on the PVA could be reacted with succinic anhydride to provide acid groups. Inks were not made from these resins. 
Because of the difficult synthesis of the PVA resins and the good performance of the other classes of resins in this project. it was not considered worthwhile to pursue development of the PVA resins and this part of the project was therefore curtailed.

\section{Dielectric spectroscopy}

BEP is currently interested in intaglio inks for web printing. These inks must dry to the touch in a second or less.

Room temperature curing of air-dry intaglio inks takes at least hours. Clearly, the cure of a web printed intaglio ink must be carried out at as high a temperature as the paper substrate will withstand. The high temperature could be used to evaporate some solvent component(s) of the ink and achieve an apparent "fast-set". Inks can be designed which will cure relatively quickly by including more air dry arms on each molecule. The polyvinyl alcohol-based resins are examples.

\section{Dielectric spectroscopy was assessed as a means of} characterizing the rate of cure of air dry resins. A thin cell was assembled with one face being a copper electrode coated with a film of the alkyd resin to be studied. About $1 \%$ of a cobalt drier was added to the resin. A counter electrode was made from a stainless steel disc perforated to allow absorption of oxygen from the air. The dielectric spectrometer was developed by Dr. F. I. Mopsik of the NIST Polymers Division and has been commercialized by IMASS Corporation. During the cure, the glass transition temperature of the resin changes, changing ionic mobility and electrical capacitance in the resin. The results are unaffected by the opacity of the specimen, which makes the technique attractive as a possibility to study the cure of inks. Studies of cure rate and of subsequent stiffness in the cured film are important. It is especially important to have some quantitative way of measuring rapid cures under a variety of conditions if web inks are to be designed and developed.

The results in the case of air-dry resins were that electronic effects arising from ionic conductivity, probably from the carboxylic acid groups used to confer Dalmar dispersibility, overpowered any change in dielectric character and the measurements did not change enough with cure to be useful. The technique works well in the case of the epoxies because epoxies cure to form much harder films than the films obtained from air resins. Until this work, it was not known how hard a film had to be in order to be suitable for the dielectric technique. 
A problem which is found to be present in "normal" air-dry films which cure slowly at room temperature will probably also arise in the long-term durability of the printing based on fast-curing air-dry resins. The problem is that cure continues over time, up to at least two years, and the film becomes more and more brittle. A recent publication ${ }^{4}$ from ICI states that ICI has found it worthwhile in terms of long time endurance of paint films to include non-reactive low $\mathrm{Tg}$ (glass transition temperature) microgels in the formulations. The microgels act as very high molecular weight plasticizers which improve the long-term mechanical properties of the films.

An articles on reactive microgels was the subject of the 1988 Joseph J. Mattiello lecture. Their importance is that the gel particles consist of very large molecules which cure very quickly but that the molecules do not extend very far into the surrounding liquid when the molecules are in "solution" or dispersion in a solvent and thus the resins are not very viscous. In this sense, they are related to latexes and emulsions.

These microgels should be considered as possible components of intaglio inks to improve durability, especially with respect to embrittlement and the subsequent "flaking" of the printed ink.

\section{References}

1. "Resins for Surface Coatings", P. Olding and G. Hayward, Eds., in 3 volumes, SITA Technology, London, (1987).

2. B. Dickens, B.J. Bauer, W.R. Blair and E.J. Parks, "Design and synthesis of prototype air-dry resins for use in BEP intaglio ink vehicles", NISTIR 89-4110, September 1989.

3. "District of Columbia Air Quality Control Regulations, Effective March 15, 1985" Environment Reporter, Bureau of National Affairs, publ., 341:0501 Appendix 3.

4. C.W. Bromley, "Nonaqueous dispersion polymer microgels as film formers in architectural paints", J. Coatings Technol., 61 , \#768, 39-43, January 1989

5. W.E. Funke, "Microgels - Intramolecularly Crosslinked Macromolecules: Potent Components of Organic Coatings", $\mathrm{J}$. Coating Technol., 60, \#767, 69-76, December 1988 
Appendix: Synthesis of pentaerythritol alkyd 61

Initially, a relatively small quantity of alkyd 61 was prepared using the following proportions of reactants.

\begin{tabular}{lr} 
Linseed oil fatty acids & $81.9 \mathrm{~g}$ \\
Sebacic acid & $21.8 \mathrm{~g}$ \\
Pentaerythritol & $26.7 \mathrm{~g}$ \\
Succinic anhydride & $19.6 \mathrm{~g}$ \\
\hline Total reactants & $-150.0 \mathrm{~g}$ \\
Solvent: Xylenes & $13.0 \mathrm{~g}$
\end{tabular}

Reaction of this quantity of material resulted in the recovery of 130 to $135 \mathrm{~g}$ of resin to give a yield of 86 to $90 \% .9 .2 \mathrm{~mL}$ of water were formed.

When a larger amount (approximately $1000 \mathrm{~g}$ ) of resin 61 was requested, the quantities of all starting materials used in the initial synthesis were increased by a factor of 2.5 times and synthesis reactions were carried out using the proportions of compounds below:

\begin{tabular}{|c|c|}
\hline $\begin{array}{l}\text { Linseed oil fatty acids } \\
\text { Sebacic acid } \\
\text { Pentaerythritol } \\
\text { Succinic anhydride }\end{array}$ & $\begin{array}{r}204.75 \mathrm{~g} \\
54.50 \mathrm{~g} \\
66.75 \mathrm{~g} \\
49.00 \mathrm{~g}\end{array}$ \\
\hline$------------1---1$ & \\
\hline $\begin{array}{l}\text { otal reactants } \\
\text { olvent: Xylenes }\end{array}$ & $\begin{array}{r}375.00 \mathrm{~g} \\
32.60 \mathrm{~g}\end{array}$ \\
\hline
\end{tabular}

Reaction of this quantity of material resulted in the recovery of 345 to $360 \mathrm{~g}$ of resin to give a yield of 92 to $96 \%$. $23 \mathrm{~mL}$ of water were formed.

The quantities of reactants used for synthesis of resin \# 61 were again increased to meet a request for approximately $4000 \mathrm{~g}$ of the resin. The following proportions of compounds, five times the original amounts, were used:

\begin{tabular}{lr} 
Linseed oil fatty acids & $409.5 \mathrm{~g}$ \\
Sebacic acid & $109.0 \mathrm{~g}$ \\
Pentaerythritol & $133.5 \mathrm{~g}$ \\
Succinic anhydride & $98.0 \mathrm{~g}$ \\
\hline Total reactants & $--1-0-0$ \\
Solvent: Xylenes & $750.0 \mathrm{~g}$ \\
\end{tabular}

Reaction of this quantity of material resulted in the recovery of 693 to $722 \mathrm{~g}$ of resin, for a yield of 92 to $96 \%$. $46 \mathrm{~mL}$ of water were formed. Solvent remaining in the finished resin averaged $2.2 \%$ by weight. 
The sebacic acid and succinic anhydride used in the synthesis of alkyd 61 were obtained from Aldrich Chemical Co.*, 940 West St. Paul Ave., Milwaukee, Wisconsin 53233, catalog numbers S175-2 and 13,441-4 respectively. The linseed oil fatty acids were obtained from spencer-Kellogg Products*, NL Chemicals, Wyckoff Mills Rd, P.O.Box 700, Hightown, NJ 08520. The pentaerythritol (technical grade) was obtained from Perstorp* , 600 Matzinger Road, Toledo, Ohio 43612. Mixed Xylenes were obtained from Fisher Scientific Co.*, Fair Lawn, NJ 07410. A silicone high vacuum grease (Dow Corning Corp.*, Midland, Michigan 48640) was used in assembling the ground glass joints of the apparatus. All glassware was cleaned between synthesis reactions in a base bath made by dissolving one pound of potassium hydroxide pellets in five gallons of $95 \%$ ethyl alcohol.

\section{Apparatus}

The same glassware configuration was used for the synthesis of alkyd 61 regardless of the volume of reactants. The only variation was in the physical size of the round bottom flask in which the reactants were mixed. As the amount of reactants was scaled up, flask volume was increased from $500 \mathrm{~mL}$ to $1000 \mathrm{~mL}$ and finally to $2000 \mathrm{~mL}$.

The glassware consisted of the following components. A three neck round bottomed flask held the reactants and solvent. A thermometer was positioned by appropriate ground glass adapters so that it was immersed in the reactants. A Dean stark distillation assembly topped with a Friedrich condenser was attached to the round bottomed flask via ground glass joints. The condenser was attached to a nitrogen bubbler so that a positive nitrogen gas pressure could be maintained in the reaction apparatus throughout the synthesis. The unused flask neck was capped with a ground glass plug. The round bottomed flask was heated by an electric heating mantle controlled with a variac and an automatic temperature sensor (Therm-o-watch*, model L7-800, IRI, Cheltenham, PA 19012) attached to the thermometer immersed in the reactants. The flask contents were stirred by a teflon covered magnetic stirring bar driven by a stirring motor located below the flask.

\section{Procedure}

Linseed oil fatty acids, sebacic acid, pentaerythritol and xylene were weighed into the round bottomed flask and the glassware was assembled. The side arm of the Dean stark trap was filled with xylene before the Friedrich condenser was attached. The stirring action was started and the glassware was purged with 
nitrogen gas. Purging was continued for 5 minutes with $500 \mathrm{~mL}$ and $1000 \mathrm{~mL}$ flasks and for 10 minutes with the $2000 \mathrm{~mL}$ flask. Following the nitrogen purge, heating was started. At 170 to $175^{\circ} \mathrm{C}$, the $x y l e n e$ refluxed and water liberated by the esterification reaction displaced xylene from the Dean stark trap.

The theoretical total amount of water liberated by the esterification reaction was calculated for each of the three volumes of reactants used. As the amount of water collected approached the theoretical total, a sample of the reaction mixture in the flask was removed and titrated with an isopropanol solution of potassium hydroxide with $0.3 \%$ thymol blue indicator in isopropanol to determine the acid number of the mixture.

Heating was continued until the acid number reached a value of 10 to 13. Heating was then stopped and the reaction mixture cooled to 135 to $140^{\circ} \mathrm{C}$. At this temperature, succinic anhydride was added and heat gently applied to raise and maintain the temperature of the reactants at $180^{\circ} \mathrm{C}$. Heating was generally stopped at approximately $160^{\circ} \mathrm{C}$ to prevent overshooting the $180^{\circ} \mathrm{C}$ temperature. The $180^{\circ} \mathrm{C}$ temperature was maintained for 30 minutes, after which time the reaction was considered complete. The finished resin was cooled and transferred to storage jars. The final acid number was 85 to $100 \mathrm{mg} \mathrm{KOH} / \mathrm{g}$ resin.

* DISCLAIMER: Materials and equipment mentioned in this report are identified to document the procedures. Such identification is not to be taken as endorsement by NIST. 



\section{BIBLIOGRAPHIC DATA SHEET}

family of air-dry intaglio resin alkyds have been developed and industrial quantities have een synthesized for press trials. The alkyds are based on pentaerythritol, sebacic acid, inse sd si? fatty acids and succinic anhydride. In laboratory resta, intaglio inks made rom these resins have washed well and cured to satisfactorily resistant films.

second series of resins has also been developed. Satisfactory washing and solvent esistance are obtained in laboratory tests. It may be worthwhile to synthesize bulk uantities of this series for press trials. These resins are based on tripentaerythritol, inseed oil fatty acids and succinic anhydride.

third series of resins based on poly(vinyl alcohol) was synthesized but did not appear to ie satisfactory for industrial development.

'he temperature program in the alkyd synthesis is important. The influence of temperature las been studied so that satisfactory resins can be made industrially in minimum time.

IEY WOROS (6 TO 12 ENTRIES; ALPHABETICAL OADER; CAPITAUZE ONLY PAOPER MAMES; AND SEPARATE KEY WOADS BY SEMICOLONS) zir-dry; alkyd; intaglio ink resins

FOR OfFICIAL DISTAIBUTION. DO MOT RELEASE TO MATIONAL TECHMICAL INFOAMATION SERVICE (NTIS).

ORDEA FAOM SUPEAINTENDENT OF DOCUMENTS, U.S. OOVERMMENT PAINTINO OFFICE, 


